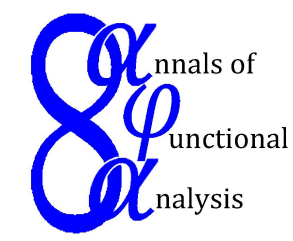

Ann. Funct. Anal. 6 (2015), no. 1, 267-278

http://doi.org/10.15352/afa/06-1-20

ISSN: 2008-8752 (electronic)

http://projecteuclid.org/afa

\title{
ON GENERALIZED BECKNER'S INEQUALITY
}

\author{
KICHI-SUKE SAITO*1 AND RYOTARO TANAKA ${ }^{2}$
}

Communicated by Y. Seo

\begin{abstract}
In this paper, we present generalizations of Beckner's inequality by using symmetric absolute normalized norms on $\mathbb{R}^{2}$.
\end{abstract}

\section{INTRODUCTION}

The aim of this paper is to present generalizations of the following inequality.

Theorem. Let $1<p \leq q<\infty$, and let $\gamma_{p, q}=\sqrt{(p-1) /(q-1)}$. Then the inequality

$$
\left(\frac{\left|u+\gamma_{p, q} v\right|^{q}+\left|u-\gamma_{p, q} v\right|^{q}}{2}\right)^{1 / q} \leq\left(\frac{|u+v|^{p}+|u-v|^{p}}{2}\right)^{1 / p}
$$

holds for all $u, v \in \mathbb{R}$.

This was shown in 1975 by Beckner [1]. It is known that $\gamma_{p, q}$ in Theorem 1 is the best constant for the inequality, that is, if $\gamma \in[0,1]$ and the inequality

$$
\left(\frac{|u+\gamma v|^{q}+|u-\gamma v|^{q}}{2}\right)^{1 / q} \leq\left(\frac{|u+v|^{p}+|u-v|^{p}}{2}\right)^{1 / p}
$$

holds for all $u, v \in \mathbb{R}$, then we have $\gamma \leq \gamma_{p, q}$. In [9], we constructed an elementary proof of these facts.

To generalize Beckner's inequality, we make use of symmetric absolute normalized norms on $\mathbb{R}^{2}$. A norm $\|\cdot\|$ on $\mathbb{R}^{2}$ is said to be absolute if $\|(x, y)\|=\|(|x|,|y|)\|$ for all $(x, y) \in \mathbb{R}^{2}$, normalized if $\|(1,0)\|=\|(0,1)\|=1$, and symmetric if $\|(x, y)\|=\|(y, x)\|$. The set of all absolute normalized norms on $\mathbb{R}^{2}$ is denoted by

Date: Received: Dec. 4, 2013; Accepted: Feb. 7, 2014.

* Corresponding author.

2010 Mathematics Subject Classification. Primary 46B20; Secondary 46B99.

Key words and phrases. Beckner's inequality, symmetric absolute normalized norm, norm inequality. 
$A N_{2}$. Bonsall and Duncan [3] showed the following characterization of absolute normalized norms on $\mathbb{R}^{2}$. Namely, the set $A N_{2}$ of all absolute normalized norms on $\mathbb{R}^{2}$ is in a one-to-one correspondence with the set $\Psi_{2}$ of all convex functions $\psi$ on $[0,1]$ satisfying $\max \{1-t, t\} \leq \psi(t) \leq 1$ for all $t \in[0,1]$ (cf. [6]). The correspondence is given by the equation $\psi(t)=\|(1-t, t)\|$ for all $t \in[0,1]$. Note that the norm $\|\cdot\|_{\psi}$ associated with the function $\psi \in \Psi_{2}$ is given by

$$
\|(x, y)\|_{\psi}= \begin{cases}(|x|+|y|) \psi\left(\frac{|y|}{|x|+|y|}\right) & \text { if }(x, y) \neq(0,0) \\ 0 & \text { if }(x, y)=(0,0)\end{cases}
$$

For some other results concerning absolute normalized norms, we refer the readers to $[5,6,7,8]$. We remark that the norm $\|\cdot\| \in A N_{2}$ is symmetric if and only if $\psi(t)=\psi(1-t)$ for all $t \in[0,1]$. For example, the function $\psi_{p}$ corresponding to the $\ell_{p}$-norm $\|\cdot\|_{p}$ is given by

$$
\psi_{p}(t)= \begin{cases}\left((1-t)^{p}+t^{p}\right)^{1 / p} & \text { if } 1 \leq p<\infty \\ \max \{1-t, t\} & \text { if } p=\infty\end{cases}
$$

and satisfies $\psi_{p}(t)=\psi_{p}(1-t)$ for all $t \in[0,1]$. Let $\Psi_{2}^{S}=\left\{\psi \in \Psi_{2}: \psi(t)=\right.$ $\psi(1-t)$ for all $t \in[0,1]\}$.

Using the functions $\psi_{p}$ and $\psi_{q}$, Beckner's inequality can be viewed as follows: Let $1<p \leq q<\infty$, and let $\gamma_{p, q}=\sqrt{(p-1) /(q-1)}$. Then the inequality

$$
\frac{\left\|\left(u+\gamma_{p, q} v, u-\gamma_{p, q} v\right)\right\|_{q}}{2 \psi_{q}\left(\frac{1}{2}\right)} \leq \frac{\|(u+v, u-v)\|_{p}}{2 \psi_{p}\left(\frac{1}{2}\right)}
$$

holds for all $u, v \in \mathbb{R}$. Inspired by this observation, in this paper, we consider the following problem.

Problem 1.1. Let $\varphi, \psi \in \Psi_{2}^{S}$, and let $\gamma \in[0,1]$. When does the inequality

$$
\frac{\|(u+\gamma v, u-\gamma v)\|_{\varphi}}{2 \varphi\left(\frac{1}{2}\right)} \leq \frac{\|(u+v, u-v)\|_{\psi}}{2 \psi\left(\frac{1}{2}\right)}
$$

hold for all $u, v \in \mathbb{R}$ ?

For each $\varphi, \psi \in \Psi_{2}^{S}$, let $\Gamma(\varphi, \psi)$ be the set of all $\gamma \in[0,1]$ such that

$$
\frac{\|(u+\gamma v, u-\gamma v)\|_{\varphi}}{2 \varphi\left(\frac{1}{2}\right)} \leq \frac{\|(u+v, u-v)\|_{\psi}}{2 \psi\left(\frac{1}{2}\right)}
$$

holds for all $u, v \in \mathbb{R}$. Needless to say, the inequality is trivial if $\gamma=0$. Thus our main purpose is to clarify the condition that $\Gamma(\varphi, \psi) \neq\{0\}$.

\section{Generalizations of Beckner's inequality}

The following is an important characterization of absolute norms on $\mathbb{R}^{2}$. The proof can be found in [2, Proposition IV.1.1] (see, also [7, Lemma 4.1]).

Lemma 2.1. A norm $\|\cdot\|$ on $\mathbb{R}^{2}$ is absolute if and only if it is monotone, that is, if $\left|x_{1}\right| \leq\left|x_{2}\right|$ and $\left|y_{1}\right| \leq\left|y_{2}\right|$ then $\left\|\left(x_{1}, y_{1}\right)\right\| \leq\left\|\left(x_{2}, y_{2}\right)\right\|$.

The following lemma is a key. 
Lemma 2.2. Let $\varphi, \psi \in \Psi_{2}^{S}$, and let $\gamma \in[0,1]$. Then the following are equivalent:

(i) The inequality

$$
\frac{\|(u+\gamma v, u-\gamma v)\|_{\varphi}}{2 \varphi\left(\frac{1}{2}\right)} \leq \frac{\|(u+v, u-v)\|_{\psi}}{2 \psi\left(\frac{1}{2}\right)}
$$

holds for all $u, v \in \mathbb{R}$.

(ii) The inequality

$$
\frac{\|(1+\gamma u, 1-\gamma u)\|_{\varphi}}{2 \varphi\left(\frac{1}{2}\right)} \leq \frac{\|(1+u, 1-u)\|_{\psi}}{2 \psi\left(\frac{1}{2}\right)}
$$

holds for all $u \in[0,1]$.

Proof. It is enough to show that (ii) $\Rightarrow$ (i). Suppose that (ii) holds. We first take an arbitrary $u>1$. Then $|1 \pm \gamma u| \leq|u \pm \gamma|$, which and Lemma 2.1 imply that

$$
\begin{aligned}
\frac{\|(1+\gamma u, 1-\gamma u)\|_{\varphi}}{2 \varphi\left(\frac{1}{2}\right)} & \leq \frac{\|(u+\gamma, u-\gamma)\|_{\varphi}}{2 \varphi\left(\frac{1}{2}\right)} \\
& =\frac{u\left\|\left(1+\gamma u^{-1}, 1-\gamma u^{-1}\right)\right\|_{\varphi}}{2 \varphi\left(\frac{1}{2}\right)} \\
& \leq \frac{u\left\|\left(1+u^{-1}, 1-u^{-1}\right)\right\|_{\psi}}{2 \psi\left(\frac{1}{2}\right)} \\
& =\frac{\|(1+u, 1-u)\|_{\psi}}{2 \psi\left(\frac{1}{2}\right)}
\end{aligned}
$$

Next, let $u \leq 0$. Since $\varphi, \psi \in \Psi_{2}^{S}$, the assumption and above inequality show that

$$
\begin{aligned}
\frac{\|(1+\gamma u, 1-\gamma u)\|_{\varphi}}{2 \varphi\left(\frac{1}{2}\right)} & =\frac{\|(1-\gamma u, 1+\gamma u)\|_{\varphi}}{2 \varphi\left(\frac{1}{2}\right)} \\
& \leq \frac{\|(1-u, 1+u)\|_{\psi}}{2 \psi\left(\frac{1}{2}\right)} \\
& =\frac{\|(1+u, 1-u)\|_{\psi}}{2 \psi\left(\frac{1}{2}\right)} .
\end{aligned}
$$

Thus the inequality

$$
\frac{\|(1+\gamma u, 1-\gamma u)\|_{\varphi}}{2 \varphi\left(\frac{1}{2}\right)} \leq \frac{\|(1+u, 1-u)\|_{\psi}}{2 \psi\left(\frac{1}{2}\right)}
$$

holds for all $u \in \mathbb{R}$.

Finally, take arbitrary $u, v \in \mathbb{R}$. If $u=0$, we have

$$
\frac{\|(\gamma v,-\gamma v)\|_{\varphi}}{2 \varphi\left(\frac{1}{2}\right)}=\gamma|v| \leq|v|=\frac{\|(v,-v)\|_{\psi}}{2 \psi\left(\frac{1}{2}\right)}
$$


So we assume that $u \neq 0$. Then

$$
\begin{aligned}
\frac{\|(u+\gamma v, u-\gamma v)\|_{\varphi}}{2 \varphi\left(\frac{1}{2}\right)} & =\frac{|u|\left\|\left(1+\gamma u^{-1} v, 1-\gamma u^{-1} v\right)\right\|_{\varphi}}{2 \varphi\left(\frac{1}{2}\right)} \\
& \leq \frac{|u|\left\|\left(1+u^{-1} v, 1-u^{-1} v\right)\right\|_{\psi}}{2 \psi\left(\frac{1}{2}\right)} \\
& =\frac{\|(u+v, u-v)\|_{\psi}}{2 \psi\left(\frac{1}{2}\right)} .
\end{aligned}
$$

This completes the proof.

We remark that (ii) in the preceding lemma is equivalent to the following condition:

$$
\frac{\varphi\left(\frac{1-\gamma u}{2}\right)}{\psi\left(\frac{1-u}{2}\right)} \leq \frac{\varphi\left(\frac{1}{2}\right)}{\psi\left(\frac{1}{2}\right)}
$$

for all $u \in[0,1]$. Thus it follows that

$$
\Gamma(\varphi, \psi)=\left\{\gamma \in[0,1]: \frac{\varphi\left(\frac{1-\gamma u}{2}\right)}{\psi\left(\frac{1-u}{2}\right)} \leq \frac{\varphi\left(\frac{1}{2}\right)}{\psi\left(\frac{1}{2}\right)} \text { for all } u \in[0,1]\right\} .
$$

for all $\varphi, \psi \in \Psi_{2}^{S}$. Moreover, since the function

$$
[0,1] \ni \gamma \rightarrow \frac{\varphi\left(\frac{1-\gamma u}{2}\right)}{\psi\left(\frac{1-u}{2}\right)}
$$

is continuous and convex for each fixed $u \in[0,1]$, the set $\Gamma(\varphi, \psi)$ is closed and convex. This means that $\Gamma(\varphi, \psi)$ is a subinterval of $[0,1]$. Let $\gamma_{\varphi, \psi}=\max \Gamma(\varphi, \psi)$. We note that $\gamma_{\varphi, \psi}$ is the best constant for the inequality.

In what follows, we study the condition for $\gamma_{\varphi, \psi}>0$. The following is the simplest result in this direction.

Proposition 2.3. Let $\varphi, \psi \in \Psi_{2}^{S}$. Suppose that $\varphi(t)=\varphi(1 / 2)$ on $[\delta, 1-\delta]$ for some $0 \leq \delta<1 / 2$. Then $\gamma_{\varphi, \psi}>0$.

Proof. Let $\gamma=1-2 \delta>0$. Then we have

$$
\frac{1}{2} \geq \frac{1-\gamma u}{2} \geq \frac{1-\gamma}{2}=\delta
$$

for all $u \in[0,1]$, which implies that

$$
\frac{\varphi\left(\frac{1-\gamma u}{2}\right)}{\psi\left(\frac{1-u}{2}\right)}=\frac{\varphi\left(\frac{1}{2}\right)}{\psi\left(\frac{1-u}{2}\right)} \leq \frac{\varphi\left(\frac{1}{2}\right)}{\psi\left(\frac{1}{2}\right)} .
$$

Thus $\gamma \in \Gamma(\varphi, \psi)$, and so $\gamma_{\varphi, \psi} \geq \gamma>0$.

For each $\psi \in \Psi_{2}^{S}$, remark that $\psi_{L}^{\prime}(1 / 2) \leq 0$, and that $\psi_{L}^{\prime}(1 / 2)=0$ if and only if $\psi$ is differentiable at $1 / 2$, where $\psi_{L}^{\prime}$ denotes the left derivative of $\psi$. Let $\varphi, \psi \in \Psi_{2}^{S}$. We consider the following four cases:

(I) $\varphi_{L}^{\prime}(1 / 2)=0$ and $\psi_{L}^{\prime}(1 / 2)=0$.

(II) $\varphi_{L}^{\prime}(1 / 2)=0$ and $\psi_{L}^{\prime}(1 / 2)<0$.

(III) $\varphi_{L}^{\prime}(1 / 2)<0$ and $\psi_{L}^{\prime}(1 / 2)=0$. 
(IV) $\varphi_{L}^{\prime}(1 / 2)<0$ and $\psi_{L}^{\prime}(1 / 2)<0$.

We first present the following theorem concerning cases (II), (III) and (IV).

Theorem 2.4. Let $\varphi, \psi \in \Psi_{2}^{S}$. Then the following hold:

(i) If $\varphi_{L}^{\prime}(1 / 2)=0$ and $\psi_{L}^{\prime}(1 / 2)<0$, then $\gamma_{\varphi, \psi}>0$.

(ii) If $\varphi_{L}^{\prime}(1 / 2)<0$ and $\psi_{L}^{\prime}(1 / 2)=0$, then $\gamma_{\varphi, \psi}=0$.

(iii) If $\varphi_{L}^{\prime}(1 / 2)<0$ and $\psi_{L}^{\prime}(1 / 2)<0$, then $\gamma_{\varphi, \psi}>0$.

In particular, if $\varphi_{L}^{\prime}(1 / 2)<0$ then

$$
\gamma_{\varphi, \psi} \leq \frac{\varphi\left(\frac{1}{2}\right) \psi_{L}^{\prime}\left(\frac{1}{2}\right)}{\psi\left(\frac{1}{2}\right) \varphi_{L}^{\prime}\left(\frac{1}{2}\right)}
$$

Proof. (i) We first remark that

$$
\psi(t) \geq \psi\left(\frac{1}{2}\right)-\psi_{L}^{\prime}\left(\frac{1}{2}\right)\left(\frac{1}{2}-t\right)
$$

for all $t \in[0,1 / 2]$. Since $\varphi^{\prime}(1 / 2)=0$, there exists $t_{0} \in[0,1 / 2)$ such that

$$
\frac{\varphi\left(\frac{1}{2}\right)-\varphi(t)}{\frac{1}{2}-t} \geq \frac{\psi_{L}^{\prime}\left(\frac{1}{2}\right)}{2}
$$

for all $t \in\left[t_{0}, 1 / 2\right)$, that is,

$$
\varphi(t) \leq \varphi\left(\frac{1}{2}\right)-\frac{\psi_{L}^{\prime}\left(\frac{1}{2}\right)}{2}\left(\frac{1}{2}-t\right)
$$

for all $t \in\left[t_{0}, 1 / 2\right]$. Putting $\gamma=1-2 t_{0}>0$, we have

$$
\frac{1}{2} \geq \frac{1-\gamma u}{2} \geq \frac{1-\gamma}{2}=t_{0}
$$

for $u \in[0,1]$, and hence, by an easy calculation, it follows that

$$
\frac{\varphi\left(\frac{1-\gamma u}{2}\right)}{\psi\left(\frac{1-u}{2}\right)} \leq \frac{\varphi\left(\frac{1}{2}\right)-\psi_{L}^{\prime}\left(\frac{1}{2}\right) \frac{\gamma u}{4}}{\psi\left(\frac{1}{2}\right)-\psi_{L}^{\prime}\left(\frac{1}{2}\right) \frac{u}{2}} \leq \frac{\varphi\left(\frac{1}{2}\right)-\psi_{L}^{\prime}\left(\frac{1}{2}\right) \frac{u}{4}}{\psi\left(\frac{1}{2}\right)-\psi_{L}^{\prime}\left(\frac{1}{2}\right) \frac{u}{2}} \leq \frac{\varphi\left(\frac{1}{2}\right)}{\psi\left(\frac{1}{2}\right)} .
$$

This means that $\gamma \in \Gamma(\varphi, \psi)$. Thus we obtain $\gamma_{\varphi_{\psi}} \geq \gamma>0$.

(ii) and (iii): Assume that $\varphi_{L}^{\prime}(1 / 2)<0$. Put

$$
k_{0}=\frac{\varphi\left(\frac{1}{2}\right) \psi_{L}^{\prime}\left(\frac{1}{2}\right)}{\psi\left(\frac{1}{2}\right) \varphi_{L}^{\prime}\left(\frac{1}{2}\right)}
$$

We first show the inequality $\gamma_{\varphi, \psi} \leq k_{0}$. Suppose that $k_{0}<1$, and that $k_{0}<\gamma \leq 1$. Since

$$
\frac{\gamma \psi\left(\frac{1}{2}\right) \varphi_{L}^{\prime}\left(\frac{1}{2}\right)}{\varphi\left(\frac{1}{2}\right)}<\psi_{L}^{\prime}\left(\frac{1}{2}\right)
$$

there exists $t_{0} \in[0,1 / 2)$ such that $t \in\left[t_{0}, 1 / 2\right)$ implies

$$
\frac{\psi\left(\frac{1}{2}\right)-\psi(t)}{\frac{1}{2}-t}>\frac{\gamma \psi\left(\frac{1}{2}\right) \varphi_{L}^{\prime}\left(\frac{1}{2}\right)}{\varphi\left(\frac{1}{2}\right)}
$$


that is,

$$
\psi(t)<\psi\left(\frac{1}{2}\right)-\frac{\gamma \psi\left(\frac{1}{2}\right) \varphi_{L}^{\prime}\left(\frac{1}{2}\right)}{\varphi\left(\frac{1}{2}\right)}\left(\frac{1}{2}-t\right)
$$

for all $t \in\left[t_{0}, 1 / 2\right)$. On the other hand, since

$$
\varphi(t) \geq \varphi\left(\frac{1}{2}\right)-\varphi_{L}^{\prime}\left(\frac{1}{2}\right)\left(\frac{1}{2}-t\right)
$$

for all $t \in[0,1 / 2]$, putting $u_{0}=1-2 t_{0}$, we have

$$
\begin{aligned}
\psi\left(\frac{1-u_{0}}{2}\right)=\psi\left(t_{0}\right) & <\frac{\psi\left(\frac{1}{2}\right)}{\varphi\left(\frac{1}{2}\right)}\left(\varphi\left(\frac{1}{2}\right)-\gamma \varphi_{L}^{\prime}\left(\frac{1}{2}\right)\left(\frac{1}{2}-t_{0}\right)\right) \\
& =\frac{\psi\left(\frac{1}{2}\right)}{\varphi\left(\frac{1}{2}\right)}\left(\varphi\left(\frac{1}{2}\right)-\frac{\gamma u_{0}}{2} \varphi_{L}^{\prime}\left(\frac{1}{2}\right)\right)
\end{aligned}
$$

and

$$
\varphi\left(\frac{1-\gamma u_{0}}{2}\right) \geq \varphi\left(\frac{1}{2}\right)-\frac{\gamma u_{0}}{2} \varphi_{L}^{\prime}\left(\frac{1}{2}\right)
$$

These imply that

$$
\frac{\varphi\left(\frac{1-\gamma u_{0}}{2}\right)}{\psi\left(\frac{1-u_{0}}{2}\right)}>\frac{\varphi\left(\frac{1}{2}\right)}{\psi\left(\frac{1}{2}\right)},
$$

which shows $\gamma \notin \Gamma(\varphi, \psi)$. Thus we obtain $\gamma_{\varphi, \psi} \leq k_{0}$, which also proves (ii).

In the case of (iii), we have $k_{0}>0$. Take an arbitrary $\gamma$ satisfying $0<\gamma<$ $\min \left\{k_{0}, 1\right\}$. Since

$$
\varphi_{L}^{\prime}\left(\frac{1}{2}\right)>\frac{\varphi\left(\frac{1}{2}\right) \psi_{L}^{\prime}\left(\frac{1}{2}\right)}{\gamma \psi\left(\frac{1}{2}\right)},
$$

there exists $t_{0} \in[0,1 / 2)$ such that $t \in\left[t_{0}, 1 / 2\right)$ implies

$$
\frac{\varphi\left(\frac{1}{2}\right)-\varphi(t)}{\frac{1}{2}-t} \geq \frac{\varphi\left(\frac{1}{2}\right) \psi_{L}^{\prime}\left(\frac{1}{2}\right)}{\gamma \psi\left(\frac{1}{2}\right)}
$$

that is,

$$
\varphi(t) \leq \varphi\left(\frac{1}{2}\right)-\frac{\varphi\left(\frac{1}{2}\right) \psi_{L}^{\prime}\left(\frac{1}{2}\right)}{\gamma \psi\left(\frac{1}{2}\right)}\left(\frac{1}{2}-t\right)
$$

for all $t \in\left[t_{0}, 1 / 2\right)$. Moreover, we note that

$$
\psi(t) \geq \psi\left(\frac{1}{2}\right)-\psi_{L}^{\prime}\left(\frac{1}{2}\right)\left(\frac{1}{2}-t\right)
$$

for all $t \in[0,1 / 2]$. Now, putting $\gamma_{0}=\min \left\{1-2 t_{0}, \gamma\right\}>0$, we have

$$
\frac{1}{2} \geq \frac{1-\gamma_{0} u}{2} \geq \frac{1-\gamma_{0}}{2} \geq t_{0}
$$


for all $u \in[0,1]$, which implies that

$$
\begin{aligned}
\varphi\left(\frac{1-\gamma_{0} u}{2}\right) & \leq \varphi\left(\frac{1}{2}\right)-\frac{\gamma_{0} u \varphi\left(\frac{1}{2}\right) \psi_{L}^{\prime}\left(\frac{1}{2}\right)}{2 \gamma \psi\left(\frac{1}{2}\right)} \\
& =\frac{\varphi\left(\frac{1}{2}\right)}{\psi\left(\frac{1}{2}\right)}\left(\psi\left(\frac{1}{2}\right)-\frac{\gamma_{0} u}{2 \gamma} \psi_{L}^{\prime}\left(\frac{1}{2}\right)\right) \\
& \leq \frac{\varphi\left(\frac{1}{2}\right)}{\psi\left(\frac{1}{2}\right)}\left(\psi\left(\frac{1}{2}\right)-\frac{u}{2} \psi_{L}^{\prime}\left(\frac{1}{2}\right)\right) .
\end{aligned}
$$

Then, it follows from

$$
\psi\left(\frac{1-u}{2}\right) \geq \psi\left(\frac{1}{2}\right)-\frac{u}{2} \psi_{L}^{\prime}\left(\frac{1}{2}\right)
$$

that

$$
\frac{\varphi\left(\frac{1-\gamma_{0} u}{2}\right)}{\psi\left(\frac{1-u}{2}\right)} \leq \frac{\varphi\left(\frac{1}{2}\right)}{\psi\left(\frac{1}{2}\right)}
$$

for all $u \in[0,1]$. This shows $\gamma_{0} \in \Gamma(\varphi, \psi)$, and so we have $\gamma_{\varphi, \psi} \geq \gamma_{0}>0$.

The following is an application of Theorem 2.4.

Example 2.5. For each $\alpha \in(1 / 2,1)$, let $\psi_{\alpha}$ be an element of $\Psi_{2}^{S}$ defined by

$$
\psi_{\alpha}(t)= \begin{cases}1+2(\alpha-1) t & \text { if } t \in[0,1 / 2] \\ 2 \alpha-1+2(1-\alpha) t & \text { if } t \in[1 / 2,1]\end{cases}
$$

Suppose that $\alpha, \beta \in(1 / 2,1)$, and that $\alpha \leq \beta$. Then

$$
k_{0}=\frac{\psi_{\alpha}\left(\frac{1}{2}\right)\left(\psi_{\beta}\right)_{L}^{\prime}\left(\frac{1}{2}\right)}{\psi_{\beta}\left(\frac{1}{2}\right)\left(\psi_{\alpha}\right)_{L}^{\prime}\left(\frac{1}{2}\right)}=\frac{\alpha(1-\beta)}{\beta(1-\alpha)} \leq 1 .
$$

On the other hand, for each $u \in[0,1]$, we have

$$
\begin{aligned}
\psi_{\alpha}\left(\frac{1-k_{0} u}{2}\right) & =1+(\alpha-1)\left(1-k_{0} u\right) \\
& =\alpha-(\alpha-1) k_{0} u \\
& =\alpha-\frac{\alpha(1-\beta)}{\beta} u \\
& =\frac{\alpha}{\beta}(\beta-(1-\beta) u) \\
& =\frac{\alpha}{\beta}(1+(\beta-1)(1-u)) \\
& =\frac{\alpha}{\beta} \psi_{\beta}\left(\frac{1-u}{2}\right) .
\end{aligned}
$$

Thus $k_{0} \in \Gamma\left(\psi_{\alpha}, \psi_{\beta}\right)$, which and Theorem 2.4 imply that

$$
\gamma_{\psi_{\alpha}, \psi_{\beta}}=k_{0}=\frac{\alpha(1-\beta)}{\beta(1-\alpha)} .
$$


Theorem 2.4 clarifies the relationship between $\gamma_{\varphi, \psi}$ and cases (II), (III) or (IV). However, we have no information about (I). So we next consider several special subcases of (I).

Let $\varphi, \psi \in \Psi_{2}^{S}$. Suppose that the second derivatives $\varphi^{\prime \prime}$ and $\psi^{\prime \prime}$ are continuous on $(\delta, 1-\delta)$ for some $0 \leq \delta<1 / 2$. Then we remark that $\varphi^{\prime \prime}(1 / 2) \geq 0$ and $\psi^{\prime \prime}(1 / 2) \geq 0$ by convexity. This allows us to consider the following four subcases of $(\mathrm{I})$ :

(I-a) $\varphi^{\prime \prime}(1 / 2)=0$ and $\psi^{\prime \prime}(1 / 2)=0$.

(I-b) $\varphi^{\prime \prime}(1 / 2)=0$ and $\psi^{\prime \prime}(1 / 2)>0$.

(I-c) $\varphi^{\prime \prime}(1 / 2)>0$ and $\psi^{\prime \prime}(1 / 2)=0$.

(I-d) $\varphi^{\prime \prime}(1 / 2)>0$ and $\psi^{\prime \prime}(1 / 2)>0$.

Here we do not consider the case (I-a) because of its complexity. For cases (I-b), (I-c) and (I-d), we have the following result.

Theorem 2.6. Let $\varphi, \psi \in \Psi_{2}^{S}$. Suppose that the second derivatives $\varphi^{\prime \prime}$ and $\psi^{\prime \prime}$ are continuous on $(\delta, 1-\delta)$ for some $0 \leq \delta<1 / 2$. Then the following hold:

(i) If $\varphi^{\prime \prime}(1 / 2)=0$ and $\psi^{\prime \prime}(1 / 2)>0$, then $\gamma_{\varphi, \psi}>0$.

(ii) If $\varphi^{\prime \prime}(1 / 2)>0$ and $\psi^{\prime \prime}(1 / 2)=0$, then $\gamma_{\varphi, \psi}=0$.

(iii) If $\varphi^{\prime \prime}(1 / 2)>0$ and $\psi^{\prime \prime}(1 / 2)>0$, then $\gamma_{\varphi, \psi}>0$.

In particular, if $\varphi^{\prime \prime}(1 / 2)>0$ then

$$
\gamma_{\varphi, \psi} \leq \sqrt{\frac{\varphi\left(\frac{1}{2}\right) \psi^{\prime \prime}\left(\frac{1}{2}\right)}{\psi\left(\frac{1}{2}\right) \varphi^{\prime \prime}\left(\frac{1}{2}\right)}} .
$$

Proof. (i) For each $\gamma \in(0,1]$, define the function $f_{\gamma}:[0,1-2 \delta) \rightarrow \mathbb{R}$ by the formula

$$
f_{\gamma}(u)=\frac{\psi\left(\frac{1-u}{2}\right)}{\psi\left(\frac{1}{2}\right)}-\frac{\varphi\left(\frac{1-\gamma u}{2}\right)}{\varphi\left(\frac{1}{2}\right)} .
$$

Then, the first and second derivative of $f_{\gamma}$ are as follows:

$$
\begin{aligned}
& f_{\gamma}^{\prime}(u)=\frac{1}{2}\left(\gamma \frac{\varphi^{\prime}\left(\frac{1-\gamma u}{2}\right)}{\varphi\left(\frac{1}{2}\right)}-\frac{\psi^{\prime}\left(\frac{1-u}{2}\right)}{\psi\left(\frac{1}{2}\right)}\right), \\
& f_{\gamma}^{\prime \prime}(u)=\frac{1}{4}\left(\frac{\psi^{\prime \prime}\left(\frac{1-u}{2}\right)}{\psi\left(\frac{1}{2}\right)}-\gamma^{2} \frac{\varphi^{\prime \prime}\left(\frac{1-\gamma u}{2}\right)}{\varphi\left(\frac{1}{2}\right)}\right) .
\end{aligned}
$$

So we have $f_{\gamma}^{\prime}(0)=0$ and

$$
f_{\gamma}^{\prime \prime}(0)=\frac{1}{4}\left(\frac{\psi^{\prime \prime}\left(\frac{1}{2}\right)}{\psi\left(\frac{1}{2}\right)}-\gamma^{2} \frac{\varphi^{\prime \prime}\left(\frac{1}{2}\right)}{\varphi\left(\frac{1}{2}\right)}\right)=\frac{\psi^{\prime \prime}\left(\frac{1}{2}\right)}{4 \psi\left(\frac{1}{2}\right)}>0 .
$$

From these facts, the function $f_{\gamma}$ is positive on the interval $\left[0, u_{0}\right]$ for some $u_{0} \in$ $(0,1]$. Let $\gamma_{0}=\gamma u_{0}>0$. Take an arbitrary $u \in[0,1]$ and put $v=u_{0} u$. Then

$$
0 \leq v \leq \min \left\{u_{0}, u\right\},
$$

and so

$$
\frac{1-u}{2} \leq \frac{1-v}{2} \leq \frac{1}{2}
$$


which implies that

$$
\psi\left(\frac{1-u}{2}\right) \geq \psi\left(\frac{1-v}{2}\right)
$$

Hence we have

$$
\begin{aligned}
f_{\gamma_{0}}(u) & =\frac{\psi\left(\frac{1-u}{2}\right)}{\psi\left(\frac{1}{2}\right)}-\frac{\varphi\left(\frac{1-\gamma_{0} u}{2}\right)}{\varphi\left(\frac{1}{2}\right)} \\
& \geq \frac{\psi\left(\frac{1-v}{2}\right)}{\psi\left(\frac{1}{2}\right)}-\frac{\varphi\left(\frac{1-\gamma v}{2}\right)}{\varphi\left(\frac{1}{2}\right)} \\
& =f_{\gamma}(v) \geq 0 .
\end{aligned}
$$

This shows that $\gamma_{0} \in \Gamma(\varphi, \psi)$. Thus $\gamma_{\varphi, \psi} \geq \gamma_{0}>0$.

We next suppose that $\varphi^{\prime \prime}(1 / 2)>0$. Put

$$
k_{0}=\sqrt{\frac{\varphi\left(\frac{1}{2}\right) \psi^{\prime \prime}\left(\frac{1}{2}\right)}{\psi\left(\frac{1}{2}\right) \varphi^{\prime \prime}\left(\frac{1}{2}\right)}} .
$$

(ii) As in the proof of (i), $f_{\gamma}^{\prime \prime}(0)<0$ if $\gamma>k_{0}$. Then it follows that $f_{\gamma}\left(u_{0}\right)<0$ for some $u_{0} \in(0,1-2 \delta)$. This means that $\gamma \notin \Gamma(\varphi, \psi)$, which shows that $\gamma_{\varphi, \psi} \leq k_{0}$. This proves (ii).

(iii) In this case, we obtain $k_{0}>0$. Moreover, for each $\gamma$ with $0<\gamma<$ $\min \left\{1, k_{0}\right\}$, we have $f_{\gamma}^{\prime \prime}(0)>0$. Hence the function $f_{\gamma}$ is positive on some nontrivial interval $\left[0, u_{0}\right]$. Finally, we obtain $\gamma_{\varphi, \psi}>0$ by an argument similar to that in the first paragraph.

Remark 2.7. We remark that

$$
\sqrt{\frac{\psi_{q}\left(\frac{1}{2}\right) \psi_{p}^{\prime \prime}\left(\frac{1}{2}\right)}{\psi_{p}\left(\frac{1}{2}\right) \psi_{q}^{\prime \prime}\left(\frac{1}{2}\right)}}=\sqrt{\frac{p-1}{q-1}}=\gamma_{p, q},
$$

where $\gamma_{p, q}$ is the best constant for Beckner's inequality. This gives another aspect of the value $\gamma_{p, q}$.

We next consider the duality of the Beckner type inequality. Then we need the following lemma.

Lemma 2.8. Suppose that $\varphi, \psi \in \Psi_{2}^{S}$. For each $\gamma \in[0,1]$, let

$$
A_{\gamma}=\left(\begin{array}{cc}
1+\gamma & 1-\gamma \\
1-\gamma & 1+\gamma
\end{array}\right)
$$

Then $\gamma \in \Gamma(\varphi, \psi)$ if and only if

$$
\left\|A_{\gamma}:\left(\mathbb{R}^{2},\|\cdot\|_{\psi}\right) \rightarrow\left(\mathbb{R}^{2},\|\cdot\|_{\varphi}\right)\right\| \leq \frac{2 \varphi\left(\frac{1}{2}\right)}{\psi\left(\frac{1}{2}\right)} .
$$

Proof. Let $\gamma \in \Gamma(\varphi, \psi)$. Then, by Lemma 2.2,

$$
\frac{\|(u+\gamma v, u-\gamma v)\|_{\varphi}}{2 \varphi\left(\frac{1}{2}\right)} \leq \frac{\|(u+v, u-v)\|_{\psi}}{2 \psi\left(\frac{1}{2}\right)}
$$


for all $u, v \in \mathbb{R}$. Take arbitrary $u, v \in \mathbb{R}$, and put

$$
u_{1}=u+v \quad \text { and } \quad v_{1}=u-v
$$

respectively. Applying the inequality for $u_{1}$ and $v_{1}$, we obtain

$$
\frac{\|((1+\gamma) u+(1-\gamma) v,(1-\gamma) u+(1+\gamma) v)\|_{\varphi}}{2 \varphi\left(\frac{1}{2}\right)} \leq \frac{\|(2 u, 2 v)\|_{\psi}}{2 \psi\left(\frac{1}{2}\right)}
$$

that is,

$$
\left\|A_{\gamma}(u, v)\right\|_{\varphi} \leq \frac{2 \varphi\left(\frac{1}{2}\right)}{\psi\left(\frac{1}{2}\right)}\|(u, v)\|_{\psi}
$$

Thus we have

$$
\left\|A:\left(\mathbb{R}^{2},\|\cdot\|_{\psi}\right) \rightarrow\left(\mathbb{R}^{2},\|\cdot\|_{\varphi}\right)\right\| \leq \frac{2 \varphi\left(\frac{1}{2}\right)}{\psi\left(\frac{1}{2}\right)} .
$$

Conversely, suppose that

$$
\left\|A_{\gamma}:\left(\mathbb{R}^{2},\|\cdot\|_{\psi}\right) \rightarrow\left(\mathbb{R}^{2},\|\cdot\|_{\varphi}\right)\right\| \leq \frac{2 \varphi\left(\frac{1}{2}\right)}{\psi\left(\frac{1}{2}\right)} .
$$

Let $u, v \in \mathbb{R}$. Putting

$$
u_{1}=\frac{u+v}{2} \text { and } v_{1}=\frac{u-v}{2}
$$

we have

$$
\begin{aligned}
\|(u+\gamma v, u-\gamma v)\|_{\varphi} & =\left\|\left((1+\gamma) u_{1}+(1-\gamma) v_{1},(1-\gamma) u_{1}+(1+\gamma) v_{1}\right)\right\|_{\varphi} \\
& =\left\|A_{\gamma}\left(u_{1}, v_{1}\right)\right\|_{\varphi} \\
& \leq \frac{2 \varphi\left(\frac{1}{2}\right)}{\psi\left(\frac{1}{2}\right)}\left\|\left(u_{1}, v_{1}\right)\right\|_{\psi} \\
& =\frac{\varphi\left(\frac{1}{2}\right)}{\psi\left(\frac{1}{2}\right)}\|(u+v, u-v)\|_{\psi} .
\end{aligned}
$$

Then it follows that $\gamma \in \Gamma(\varphi, \psi)$.

Let $\psi \in \Psi_{2}$, and let $\|\cdot\|_{\psi}^{*}$ be the dual norm of $\|\cdot\|_{\psi}$. Then, as in [4], one has $\|\cdot\|_{\psi}^{*} \in A N_{2}$, and the function $\psi^{*} \in \Psi_{2}$ corresponding to $\|\cdot\|_{\psi}^{*}$ is given by

$$
\psi^{*}(s)=\sup _{0 \leq t \leq 1} \frac{(1-s)(1-t)+s t}{\psi(t)}
$$

for all $s \in[0,1]$. We remark that $\psi \in \Psi_{2}^{S}$ implies $\psi^{*} \in \Psi_{2}^{S}$.

The following is our purpose.

Theorem 2.9. Let $\varphi, \psi \in \Psi_{2}^{S}$. Then $\gamma_{\varphi, \psi}=\gamma_{\psi^{*}, \varphi^{*}}$.

Proof. Since $\min _{0 \leq t \leq 1} \varphi(t)=\varphi(1 / 2)$, it follows that

$$
\varphi^{*}\left(\frac{1}{2}\right)=\sup _{0 \leq t \leq 1} \frac{(1-t) / 2+t / 2}{\varphi(t)}=\frac{1}{2 \varphi\left(\frac{1}{2}\right)} .
$$


We similarly have $\psi^{*}(1 / 2)=1 / 2 \psi(1 / 2)$, which implies that

$$
\frac{\psi^{*}\left(\frac{1}{2}\right)}{\varphi^{*}\left(\frac{1}{2}\right)}=\frac{\varphi\left(\frac{1}{2}\right)}{\psi\left(\frac{1}{2}\right)} .
$$

Now, take an arbitrary $\gamma \in[0,1]$ and define a matrix $A_{\gamma}$ as in Lemma 2.8. We remark that $A_{\gamma}^{*}=A_{\gamma}$, where $A_{\gamma}^{*}$ is the adjoint operator of $A_{\gamma}$. Hence Lemma 2.8 assures that $\gamma \in \Gamma(\varphi, \psi)$ if and only if

$$
\left\|A_{\gamma}:\left(\mathbb{R}^{2},\|\cdot\|_{\psi}\right) \rightarrow\left(\mathbb{R}^{2},\|\cdot\|_{\varphi}\right)\right\| \leq \frac{2 \varphi\left(\frac{1}{2}\right)}{\psi\left(\frac{1}{2}\right)}
$$

if and only if

$$
\left\|A_{\gamma}:\left(\mathbb{R}^{2},\|\cdot\|_{\varphi^{*}}\right) \rightarrow\left(\mathbb{R}^{2},\|\cdot\|_{\psi^{*}}\right)\right\| \leq \frac{2 \psi^{*}\left(\frac{1}{2}\right)}{\varphi^{*}\left(\frac{1}{2}\right)}
$$

if and only if $\gamma \in \Gamma\left(\psi^{*}, \varphi^{*}\right)$. Thus we have $\Gamma(\varphi, \psi)=\Gamma\left(\psi^{*}, \varphi^{*}\right)$. The proof is complete.

Finally, we extend generalized Beckner's inequality to normed spaces.

Theorem 2.10. Let $X$ be a normed space. Suppose that $\varphi, \psi \in \Psi_{2}^{S}$, and that $\gamma \in \Gamma(\varphi, \psi)$. Then the inequality

$$
\frac{\|(x+\gamma y, x-\gamma y)\|_{\varphi}}{2 \varphi\left(\frac{1}{2}\right)} \leq \frac{\|(x+y, x-y)\|_{\psi}}{2 \psi\left(\frac{1}{2}\right)}
$$

holds for all $x, y \in X$.

Proof. Take arbitrary $x, y \in X$, and put

$$
z=x+y \text { and } w=x-y
$$

respectively. We also put

$$
u=\frac{\|z\|+\|w\|}{2} \text { and } v=\frac{\|z\|-\|w\|}{2} .
$$

Then we have

$$
\begin{aligned}
\frac{\|(x+\gamma y, x-\gamma y)\|_{\varphi}}{2 \varphi\left(\frac{1}{2}\right)} & =\frac{\left\|\left(\frac{1+\gamma}{2} z+\frac{1-\gamma}{2} w, \frac{1-\gamma}{2} z+\frac{1+\gamma}{2} w\right)\right\|_{\varphi}}{2 \varphi\left(\frac{1}{2}\right)} \\
& \leq \frac{\left\|\left(\frac{1+\gamma}{2}\|z\|+\frac{1-\gamma}{2}\|w\|, \frac{1-\gamma}{2}\|z\|+\frac{1+\gamma}{2}\|w\|\right)\right\|_{\varphi}}{2 \varphi\left(\frac{1}{2}\right)} \\
& =\frac{\|(u+\gamma v, u-\gamma v)\|_{\varphi}}{2 \varphi\left(\frac{1}{2}\right)} \\
& \leq \frac{\|(u+v, u-v)\|_{\varphi}}{2 \psi\left(\frac{1}{2}\right)} \\
& =\frac{\|(x+y, x-y)\|_{\psi}}{2 \psi\left(\frac{1}{2}\right)} .
\end{aligned}
$$

This completes the proof. 


\section{REFERENCES}

1. W. Beckner, Inequalities in Fourier analysis, Ann. of Math. 102 (1975), 159-182.

2. R. Bhatia, Matrix analysis, Springer-Verlag, New York, 1997.

3. F.F. Bonsall and J. Duncan, Numerical ranges II, Cambridge University Press, Cambridge, 1973.

4. K.-I. Mitani, S. Oshiro and K.-S. Saito, Smoothness of $\psi$-direct sums of Banach spaces, Math. Inequal. Appl. 8 (2005), 147-157.

5. K.-I. Mitani, K.-S. Saito and T. Suzuki, Smoothness of absolute norms on $\mathbb{C}^{n}$, J. Convex Anal. 10 (2003), 89-107.

6. K.-S. Saito, M. Kato and Y. Takahashi, Von Neumann-Jordan constant of absolute normalized norms on $\mathbb{C}^{2}$, J. Math. Anal. Appl. 244 (2000), 515-532.

7. K.-S. Saito, M. Kato and Y. Takahashi, Absolute norms on $\mathbb{C}^{n}$, J. Math. Anal. Appl. 252 (2000), 879-905.

8. Y. Takahashi, M. Kato and K.-S. Saito, Strict convexity of absolute norms on $\mathbb{C}^{2}$ and direct sums of Banach spaces, J. Inequal. Appl. 7 (2002), 179-186.

9. R. Tanaka, K.-S. Saito and N. Komuro, Another approach to Beckner's inequality, J. Math. Inequal. 7 (2013), 543-549.

${ }^{1}$ Department of Mathematics, Faculty of Science, Nitgata University, Nitgata 950-2181, JAPAN.

E-mail address: saito@math.sc.niigata-u.ac.jp

2 Department of Mathematical Science, Graduate School of Science and TechNOLOGy, NiIgATa University, NiIgata 950-2181, JaPAN.

E-mail address: ryotarotanaka@m.sc.niigata-u.ac.jp 